\title{
USING DISTANT MONITORING METHODS FOR THE CULTIVATION OF CANNABIS
}

Jiř́ SOUČEK, Research Institute of Agricultural Engineering, p. r. i., Drnovsá 507, 161 01, Prague, Czech Republic, jiri.soucek@ff.cuni.cz (corresponding author)

Marie BJELKOVÁ, Agritec Plant Research Ltd., Zemědělská 2520/16, 787 01, Šumperk, Czech Republic

Patrik BURG, Mendel University in Brno, Valtická 337, 69144 Lednice, Faculty of Horticulture, Czech Republic, patrik.burg@mendelu.cz

Algirdas JASINSKAS, Department of Agricultural Engineering and Safety, Faculty of Engineering, Vytautas Magnus University, K. Donelaičio g. 58, 44248 Kaunas, algirdas.jasinskas@ vdu.lt

\begin{abstract}
Plant monitoring with the use of distant methods is a way of acquiring the data necessary to operate modern technological systems. The development is moving towards a wider range of observed parts of the wave spectrum. The quality of vegetation and soil monitoring devices is increasing. This is associated with a higher purchase price. Operating costs are also higher. The requirements for the processing of acquired records and their interpretation are also more demanding. The complexity of connecting the obtained data with practical applications in real operation is also increasing. On the contrary, the possibility of using simpler equipment and simple evaluation of the obtained data means lower costs and lower demands on operation and software. The importance of simple monitoring methods also lies in their higher ability to act. Monitoring can be performed at the necessary time and the information obtained can be used immediately for the implementation of agrotechnical interventions. As part of growth experiments on technical cannabis, experimental monitoring using a drone equipped with a standard RGB camera was carried out. It was verified that by employing this fairly easy method, we can differentiate various levels of nitrous fertilization, especially in the red part of the visible spectrum.
\end{abstract}

Keywords: technical cannabis, UAV, RGB, nitrous fertilization, plant monitoring, Imagej

\section{INTRODUCTION}

The research into the effect of growing conditions on plant development is a fundamental source of knowledge used in the development of agriculture and thus ensuring the nutrition of the human population. Based on the gathered information, a suitable plant variety with the correct procedures regarding the nutrition, plant protection and breeding is chosen. It is also used for choosing the correct agrotechnical procedures and helps to effectively develop agricultural machinery.

Due to the rapid progress of modern technologies, namely in the fields of precision agriculture and environmentally friendly procedures, the volume and the accuracy of the data required for both the research and practice is ever-increasing. And since the currently used methods are not capable of producing the data needed, new ones need to be implemented. In addition, standard methods of monitoring also have their drawbacks. The main issue is that their realisation is very time demanding and costly (Hanly et al., 2017). This severely limits the amount of gathered results and samples taken within one examination, which leads to an insufficient information value regarding the variability of monitored properties and their distribution in the monitored area (Hůla et al., 2001).

Plant monitoring is a basic premise of an effective cultivation of agricultural crops. Based on the observation, we can create short- and long-term production strategies (Wang et al., 2017), detect any risks caused by pests and weeds (Yuan et al., 2017) and, last but not least, the environmental threats, such as erosion (Möller et al., 2017) and soil deficiencies. Hence, farmers have been increasingly using distant methods based on taking and interpreting images of plants. They are based on the findings on the correlation of monitored properties with physical quantities, such as conductivity, capacity, colour, voltage, temperature, light intensity, etc. (Abrantes et al., 2017). There are several ways of acquiring the images, all of them having their own advantages and disadvantages.

In the case of using satellite imagery, the main advantage is that the images are usually free-to-use. However, the relatively low resolution and the need to convert them into a usable form requires an extensive knowledge and experience. There is also the impossibility to completely filter out clouds, which can partially or completely cover the monitored area in most of the observed spectra.

Another method is aerial photography. Its high imaging performance with a relatively high resolution is one of its main advantages. However, the low flexibility and high costs are a large disadvantage. Due to this, a third method, which 
employs drones (UAVs) instead of airplanes, is being used more and more frequently. The drawback in the form of a lower imaging performance is heavily outweighed by the flexibility and relatively low costs. Furthermore, the flight height of a standard drone is below the cloud layers, which means that the imaging is not affected by weather as significantly. In the case of satellite imagery, the range of captured wavelengths is determined by the instrumentation and the willingness of the satellite owner to share the images. This does not apply for the aerial and drone imagery, whose possibilities are limited only by the technical capabilities of the aircraft and the capturing device used. However, it is necessary to take the laws and regulations into account.

The main concerns in the agricultural practice are the ease of execution and the effectivity of the method. Hence, taking the pictures and the subsequent evaluation should be as simple and quick to use as possible. Due to the demanding conditions of agriculture, there is a high risk of damaging the employed devices. So, if possible, the device should be durable with reasonable purchasing and operating costs.

As it was mentioned before, indirect measuring methods are based on establishing values of easily measurable quantities. One of these quantities is colour evaluation. It is based on a widespread method of correlating colour shades to number values. This allows to establish values even for the light spectra invisible to a human eye (IR, UV), by converting the images into a visible spectrum. A comparison of sensors working with different ranges of the light spectrum used in a cultivation of rice is published in (Sun et al., 2017). However, this requires quite an extensive amount of equipment, both for capturing the imagery and the subsequent evaluation of the results. From the user's viewpoint, it is also less flexible and comfortable to use in practice.

In general, it is safe to state that it is not necessary for agricultural companies, at least for those not using modern technologies for sowing, fertilizer and pesticide application and precise cultivation methods, to measure and evaluate the properties of plants. However, from the overall agricultural development and the European policies, it is more and more evident that the push for implementing "environmentally friendly agricultural practices" is rising and in the future, it will be very difficult, if not impossible, to commercially employ instrumentation deemed as "not ecological". The second aspect favouring the monitoring of vegetation with the use of distant methods is purely technical. Evaluating the actual state of the plants, including their variability, and ensuring necessary agrotechnical measures to eliminate the identified deficiencies is only possible for plants of a smaller acreage and lower growth, generally speaking, only in the time period, when it is possible to move through the plants with a standard machinery. This is a problem for species with a higher growth, cannabis being a typical example. Majority of its varieties have a high growth and high growth rate. It is impossible to assess the entirety of the crop "from the ground". In practice, it is necessary to monitor the cannabis plants to get precise information on their current state, any pest activities and yield and damage estimates. In research, it is important to choose the correct plant variety and plan precise cultivation procedures, including the establishment of the plants, fertilization and other agrotechnical actions.

In order to further analysis of these problems, the part of a growth experiment on cultivating technical cannabis, an imaging of individual variants and a subsequent evaluation was carried out. The aim was to verify the feasibility of using a drone equipped with a standard RGB camera to capture images, which can be used for a evaluation of differences in the levels of nitrous fertilization and plant density.

\section{METHODS AND MATERIALS}

As part of growth experiments of Agritec Plant Research, s.r.o, images of parcels of technical cannabis of the Fedora variety were taken and evaluated, each parcel having $100 \mathrm{~m}^{2}$. The monitoring was carried out on the 25 th of July, with the plant age being 50 days. A different level of nitrous fertilization was applied to each parcel. In total, four levels of fertilization of plants with the same level of sowing density of 1,2 million seeds per hectare were examined.

Fertilization levels: nitrogen (Calcium Nitrate)

- N0 - unfertilized N,

- $\mathrm{N} 1$ - 30kg N.ha-1,

- $\mathrm{N} 2$ - 60kg N.ha-1

- N3 - 120kg N.ha ${ }^{-1}$.

The images were taken using a standard $4 \mathrm{~K}$ camera mounted on an unmanned four-rotor drone DJi Phantom 4 Pro V2. Due to the relatively small area of the examined parcels, only one image, containing all parcels, needed to be taken. This eliminates any inconsistencies and differences between multiple images, caused, for example, by a change in the lighting intensity. Then, a series of control images of the monitored plants with an $85 \%$ overlap was taken in case that the singular image could not be properly interpreted due to the resolution being too low.

An image containing all parcels, having an even illumination without any shadows cast by clouds and having a minimal distortion, was chosen for the evaluation (Fig. 1). The image was edited in Adobe Photoshop to correct the camera lens distortion and then processed and evaluated using a commercially free software ImageJ. First, it was divided into individual parcels, each having a resolution of 1340x830 pixels. This step eliminated the edges of the parcels with an uneven growth and the space between parcels. These modified images were then analysed by filtering various ranges of the visible light spectrum; red, blue, green and greyscale. The results of the analyses were then tabulated in MS Excel and a mean value of the monitored colour was determined for each parcel. 


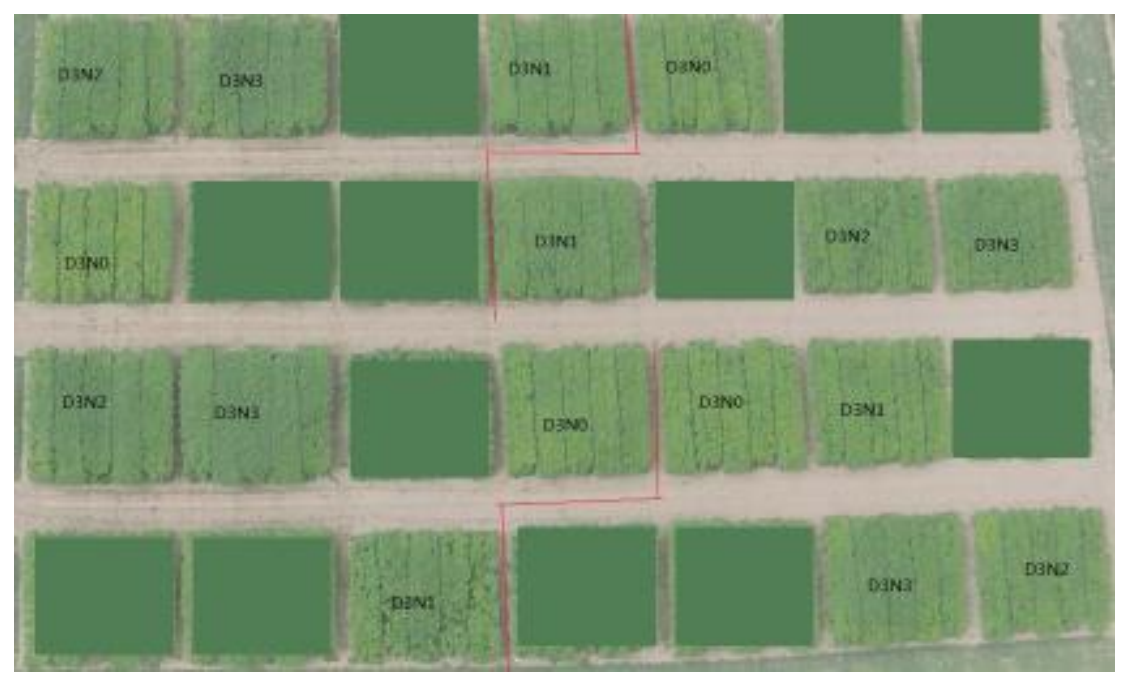

Figure 1. Monitored experimental parcels of technical cannabis

\section{RESULTS AND DISCUSSION}

The cropped images of individual experimental parcels were analytically processed in the chosen colour spectrum. Parcels with a highlighted colour temperature and indication of fertilization level and yield can be seen in Fig. 2, values in a form of histograms can be seen in Fig. 3 - Fig. 6.

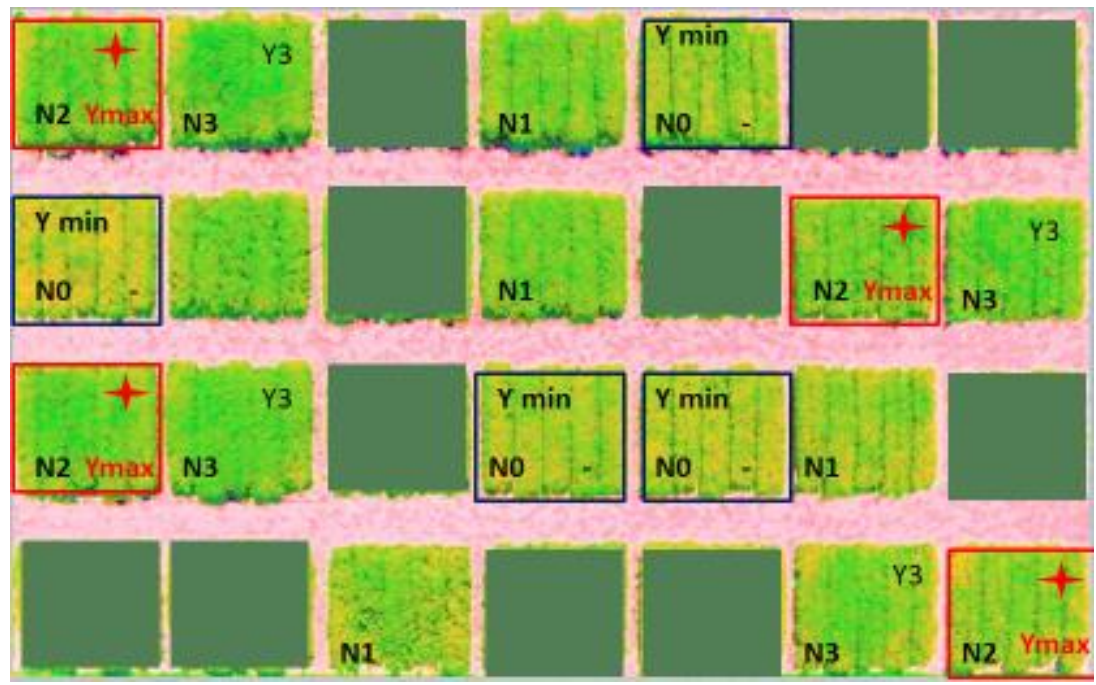

- N0 unfertilized (Y 4. min. yield)

N1 $30 \mathrm{~kg} \mathrm{~N} \cdot \mathrm{ha}^{-1}$ (Y 3. yield)

N2 60 kg N.ha ${ }^{-1}$ (Y 1. max. yield)

N3 120 kg N.ha ${ }^{-1}$ (Y 2. yield)

Figure 2. Examined parcels with a highlighted colour temperature and indication of fertilization level and yield

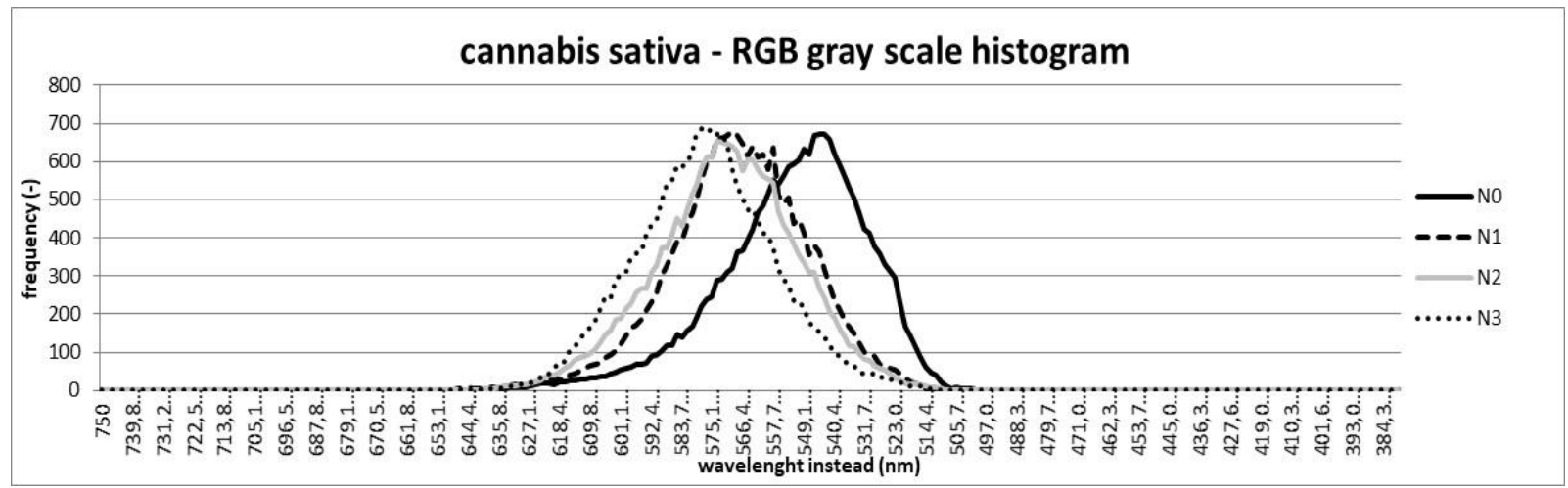

Figure 3. Analysis of RGB images of monitored variations of nitrous fertilization converted into a greyscale histogram 


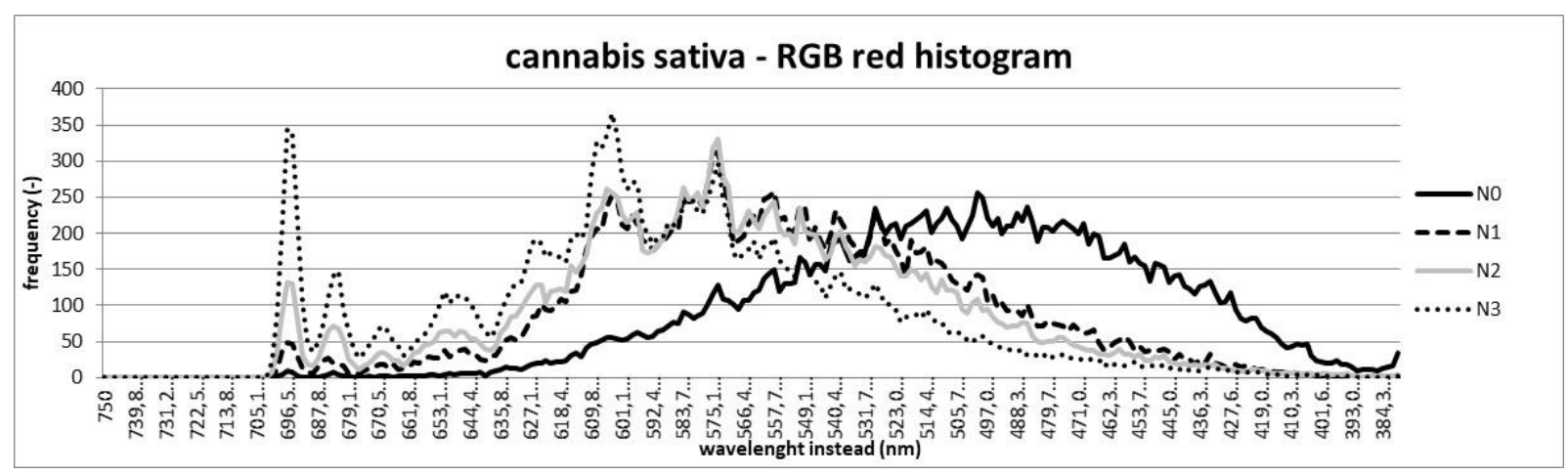

Figure 4. Analysis of RGB images of monitored variations of nitrous fertilization in a form of red histogram

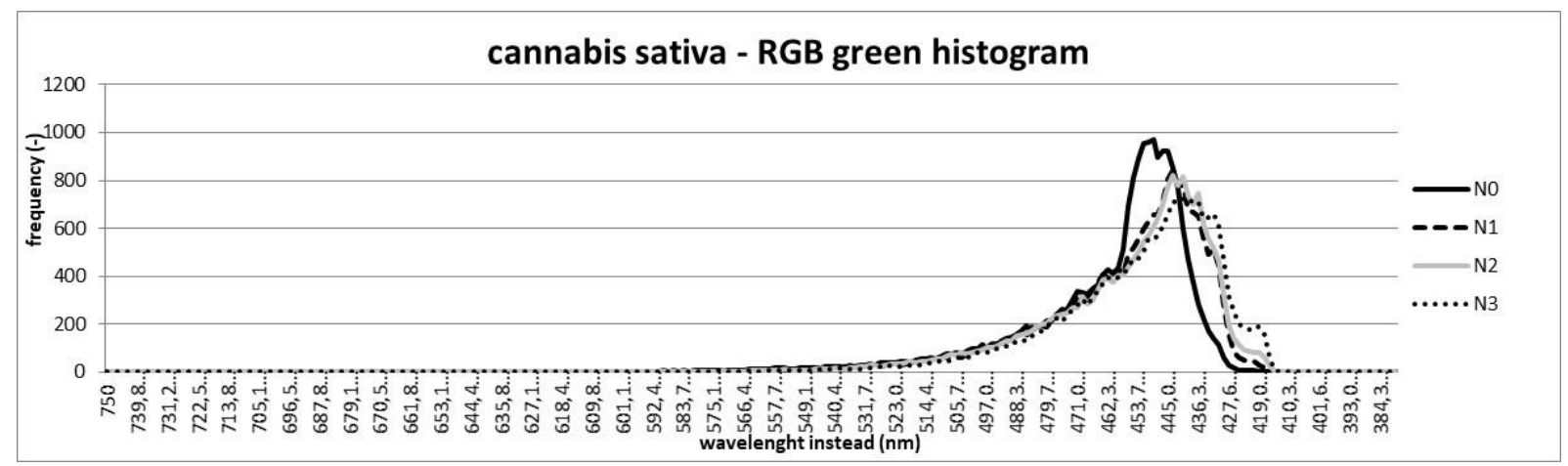

Figure 5. Analysis of RGB images of monitored variations of nitrous fertilization in a form of green histogram

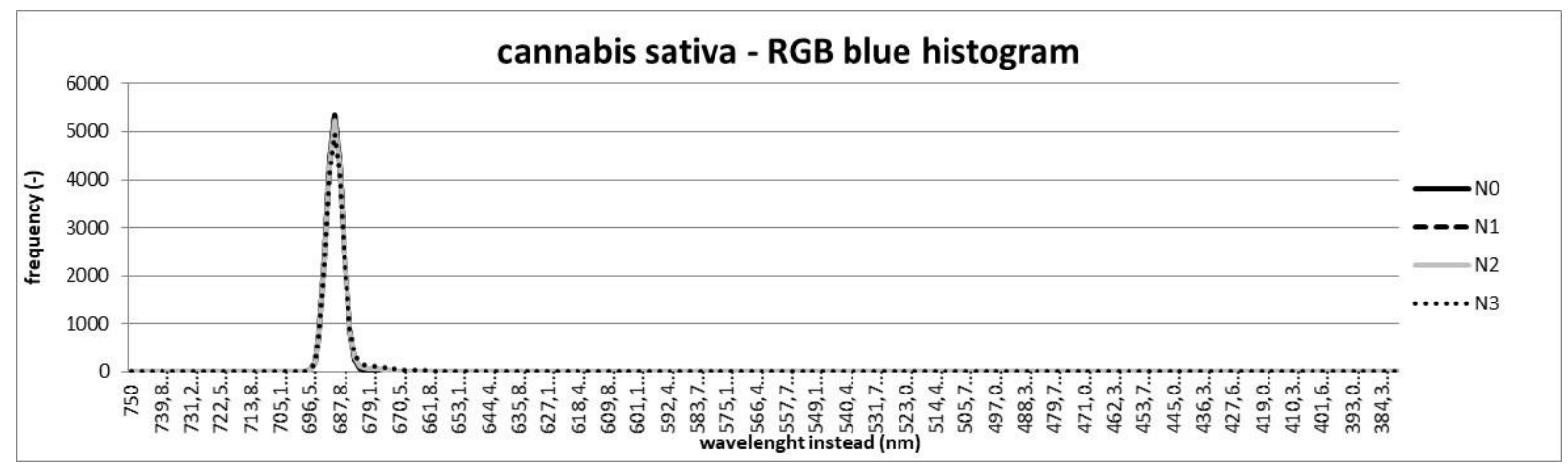

Figure 6. Analysis of RGB images of monitored variations of nitrous fertilization in a form of blue histogram

From the graphs in Fig. 3 - Fig. 6, it can clearly be seen that the differences are most distinguishable in the red part of the visible spectrum and are also sufficiently apparent in the greyscale conversion. This is in accordance with (Aitkenhead, J., et al., 2017; Szalay, K., et al., 2019). In the case of greyscale, the results differ mainly in the values of the wavelengths in which the maximum frequencies were recorded. In the case of the RGB histogram, a significant difference between the unfertilized and the fertilized parcels can be seen towards lower wavelengths. In the area of higher wavelengths $(685-700 \mathrm{~nm})$, a significant difference in the frequency of all variants can be seen, this agrees with (Jia, S., et al., 2017).

In the case of the green histogram, in the wavelength range of 445-455nm, the frequency differs only for the unfertilized variant, all other variants are almost identical. In the case of the blue histogram, there is no significant difference in the frequency of any variant. The maximum observed frequency of all variants was $690 \mathrm{~nm}$.

\section{CONCLUSIONS}

From the results, it can clearly be concluded that the indirect method of determining the effect of various nitrous fertilization levels on technical cannabis plants by an analysis of images in a visible light spectrum is feasible. Namely, images converted to a red-scale. Grey-scale also yields satisfying results. The presented method is not demanding on equipment, which means that no special sensors, expensive software or instrumentation are needed. Its main advantages are the ability to non-destructively monitor large areas of crops, the flexibility throughout the growth cycle and the ability to quickly and easily perform a measurement and evaluation. The results can be used, for example, to adjust variable fertilization rates in the field of precision agriculture. 
Acknowledgement. In this article, the results obtained within the project of the Ministry of Agriculture of the Czech Republic No. QK21010151 Obtaining vegetable oils using modern methods and the project of long time development of Research Institute of Agricultural Engineering p.r.i. no. RO0618 were used.

\section{REFERENCES}

1. Abrantes J., João L., Sérgio M., Prats A., Jacob Keizer J. J. 2017. Assessing soil water repellency spatial variability using a thermographic technique: an exploratory study using a small-scale laboratory soil flume. Geoderma, Vol. 287, pp.98-104. https://doi.org/10.1016/j.geoderma.2016.08.014

2. Aitkenhead J., Matt J., Gaskin G., Lafouge,N., Hawes C. 2017. 'PHYLIS: A low-cost portable visible range spectrometer for soil and plants. Sensors, Vol. 17 (1). 99. https://doi.org/10.3390/s17010099

3. Hanly J. A., Hedley M. J., Horne D. J. 2017. Effects of summer turnip forage cropping and pasture renewal on nitrogen and phosphorus losses in dairy farm drainage waters: a three-year field study. Agricultural water management, Vol. 181 pp. 10-17. https://doi.org/10.1016/j.agwat.2016.11.009

4. Hůla J., Kroulik M., Rybka A. 2001. The impact of the soil properties site heterogeneity upon the tillage quality. In Farm Work Science Facing Challenges of the XXI Century. Proceedings of 29th Congress of CIOSTA-CIGR V: Krakow, Poland. JUN 25-27, pp. 325-330.

5. Jia S., Shi S., Gong W., Yang J., Du L., Song, S., Chen B., Zhang Z. 2017 Evaluation of hyperspectral lidar for monitoring rice leaf nitrogen by comparison with multispectral lidar and passive spectrometer. Scientific Reports, Vol. 7, ID 40362. https://doi.org/10.1038/srep40362

6. Junker L.V., Ensminger I. 2016. Fast detection of leaf pigments and isoprenoids for ecophysiological studies, plant phenotyping and validating remote-sensing of vegetation. Physiol Plant, Vol 158, (4), pp. 369-381. https://doi.org/10.1111/ppl.12512

7. Lin Y., Zhang H., Zhang Y., Xing Ch., Bao Z. 2017. Feasibility assessment of multi-spectral satellite sensors in monitoring and discriminating wheat diseases and insects. Optik - International Journal for Light and Electron Optics, Vol. 131. pp. 598-608. https://doi.org/10.1016/j.ijleo.2016.11.206

8. Möller M., Gerstmann M. H., Gao F., Dahms T., Ch., Förster M. 2017.Coupling of phenological information and simulated vegetation index time series: Limitations and Potentials for the Assessment and Monitoring of Soil Erosion Risk. CATENA, Vol. 150. pp. 192-205. https://doi.org/10.1016/j.catena.2016.11.016

9. Szalay K., Keller B., Kovacs L., Rak R., Peterfalvi N., Sillinger F., Golub G., Kukharets S., Souček J., Jung A. 2020. Physical protection in experimental rapsberry plantation. Inmatech-Agricultural Engineering, Vol. 57 (1). pp. 115-122. https://doi.org/10.35633/INMATEH_57_12

10. Wang C, Feng M, Yang W, Ding G, Xiao L, Li G, et al. 2017. Extraction of sensitive bands for monitoring the winter wheat (triticum aestivum) growth status and yields based on the spectral reflectance. PLoS ONE, Vol. 12(1), pp. 1-16. https://doi.org/10.1371/journal.pone.0167679

11. Yang P., Byrne J. M., Yang M. 2016. Spatial variability of soil magnetic susceptibility, organic carbon and totalnitrogen from farmland in northern china. Catena, Vol. 145. pp. 92-98. https://doi.org/10.1016/j.catena.2016.05.025 